\title{
Infected Bilateral Chylothorax in a Problematic Case
}

\author{
Bulent Bakar ${ }^{\mathrm{a}, \mathrm{d}}$, Kutluk Pampal ${ }^{\mathrm{b}}$, Ismail Hakki Tekkok ${ }^{\mathrm{c}}$
}

\begin{abstract}
Within this report we describe a problematic patient who developed infected bilateral chylothorax. A 38 year-old female who had a berry aneurysm at the basilar artery was admitted to neurointensive care unit after coil embolization procedure. On third day, her chest X-ray disclosed bilateral massive pleural effusions. Bilateral intercostal drainage tube insertions were performed. The pleural fluid was clearly. Cultures of the chylous fluid revealed Acinetobacter calcoaceticus-baumanii complex. On 10th day, she expired. We conclued that chylothorax in adults may rarely occur without a solid reason; and in the treatment may also be difficult especially when it is contaminated with microorganisms.
\end{abstract}

Keywords: Aneurysm; Catheterisation; Chylothorax; Thoracic duct

\section{Introduction}

Chylothorax represent chylous liquid (ie. enriched with fat and its products) in the pleural cavity [1]. Although it is the most common aetiological factor of the pleural effusions in neonates and fetuses, in adults it is very rare with an incidence of $3-5 \%$ [2-4].

Within this report we describe a problematic patient who developed infected bilateral chylothorax after endovascular embolization of a berry aneurysm at the tip of the basilar artery.

\section{Case Report}

A 38 year-old female was admitted to emergency room with severe headache, naeusea and vomiting of sudden onset. On neurologic exam, she had neck stiffness and mild right hemiparesis. Cranial CT scan disclosed subarachnoid haemorrhage (Fisher grade IV) (Fig. 1). MR-angiography and four-vessel cerebral angiography revealed a berry aneurysm at the tip of the basilar artery (Fig. 2). She was referred for endovascular surgery. After the embolization procedure, she was admitted to the neurointensive care unit (ICU). Immediately after the general anesthetic effect, she became agitated

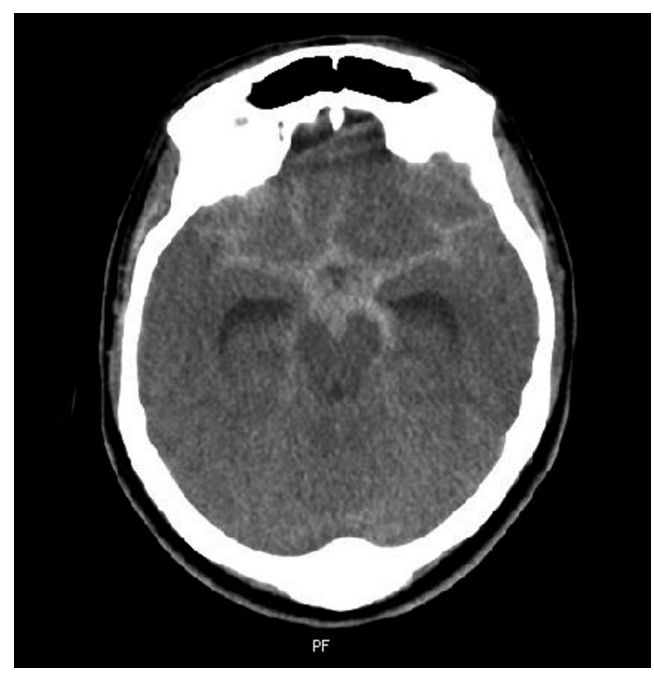

Figure 1. Cranial CT scan discloses subarachnoid haemorrhage.
Manuscript accepted for publication April 19, 2012

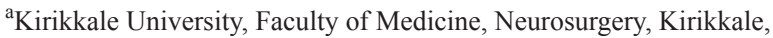
Turkey

${ }^{\mathrm{b}}$ Gazi University, Faculty of Medicine, Anaestesiology and Reanimation, Ankara, Turkey

'TOBB-ETU Hospital, Neurosurgery, Ankara, Turkey

${ }^{\mathrm{d}}$ Corresponding author: Bulent Bakar, Department of Neurosurgery, Kirikkale University, School of Medicine, 71100-Kirikkale, Turkey. Email: bulentbanrs@yahoo.com

doi: $10.4021 / \mathrm{jcs} 30 \mathrm{e}$ 


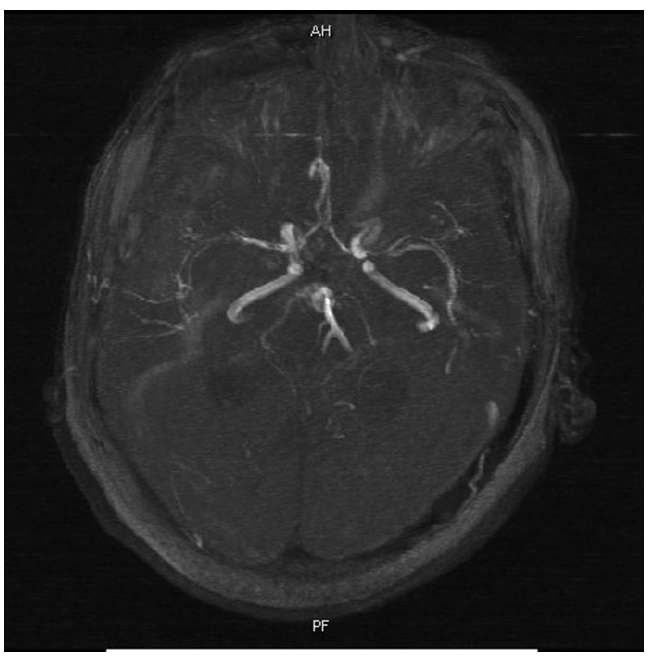

Figure 2. MR-angiography reveales a berry aneurysm at the tip of the basilar artery.

and disoriented. Considering that an unstable hemodynamic situation would be harmful for the brain, the patient was intubated and mechanically ventilated. Cranial CT scan after sedation revealed obstructive hydrocephalus (Fig. 3). A ventricular drainage catheter was inserted at right Kocher's point. As part of ICU routine a right subclavian catheter was inserted for nutrition and $3 \mathrm{H}$ (hypervolemic, hypertensive haemodilution) treatment. On third day at the ICU, the patient became pyrexic and chest X-ray revealed bilateral massive pleural effusions (Fig.4), particularly on the right side. Chest CT disclosed mediastinal enlargement (Fig. 5). Bilateral intercostal drainage tubes were inserted and approxiamtely $2000 \mathrm{~mL}$ milky fluid was drained. The biochemical analysis of chylous fluid revealed a high level of trigliseride and a low level of total cholosterol with a value of $3534 \mathrm{mg} /$ $\mathrm{dL}$ (50 - 200, normal serum ranges), $32 \mathrm{mg} / \mathrm{dL}$ (125 - 200,

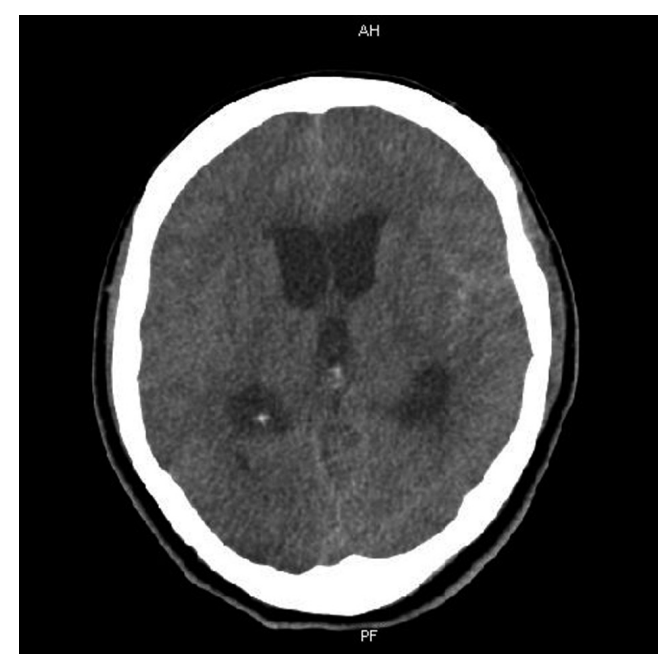

Figure 3. CT scan reveales obstructive hydrocephalus.

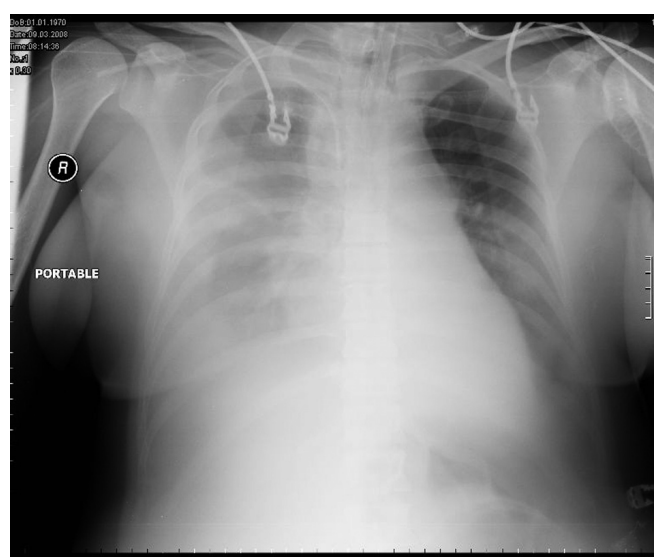

Figure 4. Chest X-ray shows bilateral massive pleural effusions.

normal serum ranges), respectively. $15 \mathrm{mg}$ twice a day subcutaneous octreotide was initiated.

Transthoracic echocardigraphy, lower limb venous doppler-US, oesophago-gastro-duodenoscopy and abdomen CT scan were normal except for bilateral surrenal hyperplasia. Urine analysis and routine blood biochemistry were normal. Elevated fibrinogen, D-dimer, Factor VIII and IX (Christmas) levels were pathological laboratory findings. Her total parenteral nutrition was continued with low chain fatty acids; and subcutaneous low molecular weight heparin were also started. Further immunological studies of serum showed antibodies for lupus anticoagulant antigen $1(+)$ (normal reference range $<4(+)$ ) and antinuclear antibodies (ANA) at a titer of 1/100 (normal reference range $<1 / 360$ ), but test for anti-ds DNA, anti-SSA/Ro, anti-SSB/La,anti-Sm and anti RNP antibodies all gave negative results. Factor V Leiden mutation analysis was normal. Culture of the chylous fluid revealed Acinetobacter calcoaceticus-baumanii complex which was resistant to piperacillin, ceftazidime, ciprofloxacine, trimethoprim-sulfamethaxozole, and tazobactam but not to amicasin, ticarcillin and imipenem, $1 \mathrm{gm}$ thrice a day meropenem and $500 \mathrm{mg}$ twice a day vancomycin intravenous (IV) was initiated. Chylous fluid cytology showed numerous

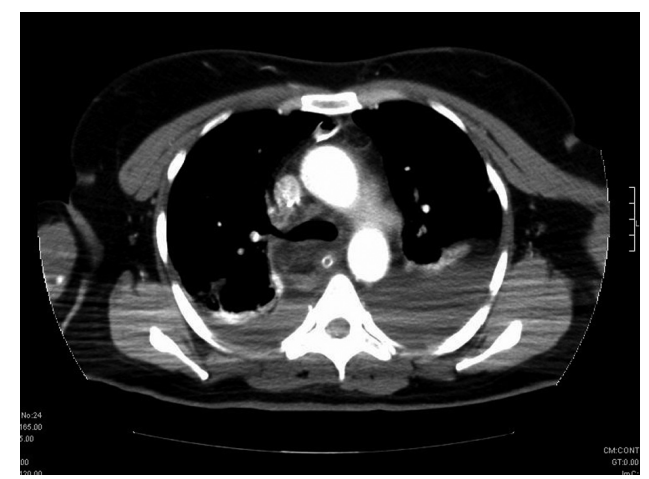

Figure 5. Chest CT discloses mediastinal enlargement. 


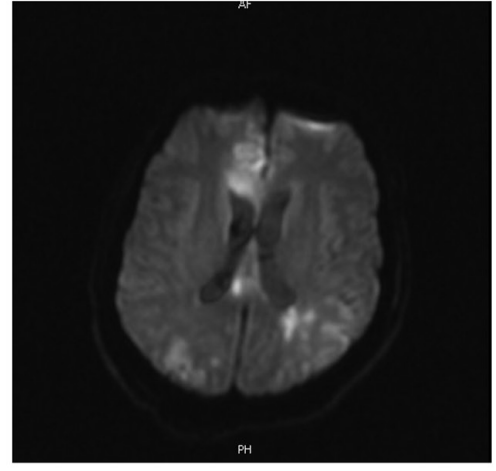

a

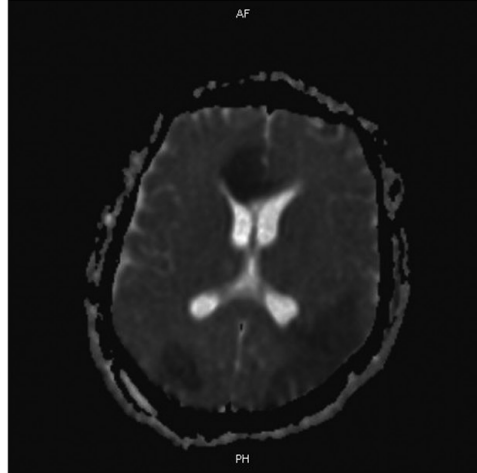

b

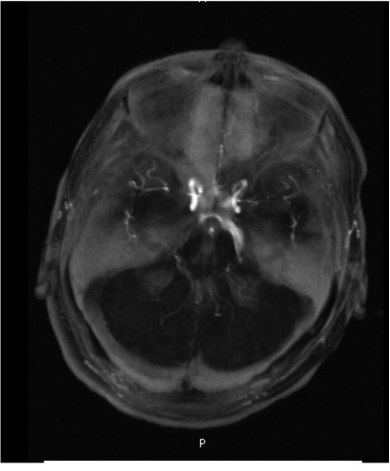

c

Figure 6. (6a and b) Diffusion and perfussion weighted cranial MR show bilateral posterior parietal, and right medial frontal hemispheric ischemia; and (6c) MR-angiography discloses vasospasm at both right anterior cerebral artery and bilateral middle cerebral arteries.

leukocytes without atypical features and its tuberculousPCR was negative. Three days later both catheters were removed. On 8th day after admission, she developed left sided hemiplegia. Diffusion and perfusion weighted cranial MR showed bilateral posterior parietal, and right medial frontal hemispheric ischemia; and MR-angiography disclosed vasospasm at right anterior cerebral and both bilateral middle cerebral arteries (Fig. 6). Chest MR revealed re-collection of the right pleural effusion, persistent mediastinal enlargement related to haemorrhage like mediastinal collection; and thrombus in the azygous vein. All main mediastinal vessels except for left inferior pulmonary artery and azygous vein were patent. Percutaneous thoracentesis was performed and $1500 \mathrm{~mL}$ chylous fluid was drained. On 9th day, her right pupil enlarged and became unresponsive to light. Cranial CT showed bilateral cerebral oedema with subfalcine herniation towards to left side (Fig. 7). An intracranial pressure (ICP)

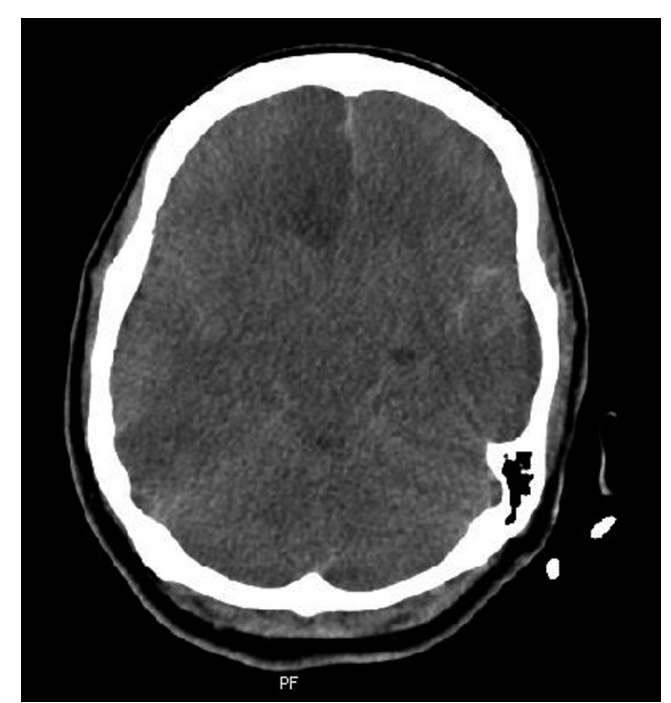

Figure 7. Cranial CT shows bilateral cerebral oedema with subfalcine herniation towards to left side. monitor probe was inserted at the left side Kocher' point, and the initial ICP was $69 \mathrm{mmHg}$. Then she underwent right fronto-temporo-parietal decompresssive craniectomy and dural release. Postoperative ICP was down to $10 \mathrm{mmHg}$. On 10th day body temperature was $40{ }^{\circ} \mathrm{C}$ and ICP levels increased to $40 \mathrm{mmHg}$. Her blood pressure level decreased steadily despite medical treatment; and she expired. The family declined a postmortem study.

\section{Discussion}

The aetiology of chylothorax in adults includes trauma to the thoracic duct (blunt or penetrating trauma), thoracic surgery, central venous catheterisation, extensive venous thrombosis in the neck, neoplasms, compression of the mediastinal lymph nodes, hepatic chirosis, idiopathic causes (such as Down's syndrome), some connective tissue diseases (such as Behçet's disease and systemic lupus erythomatosus (SLE)), infectious diseases (such as flariasis, tuberculosis and histoplasmosis), lymphangioleiomyomatosis, and congestive heart failure. If there is no history of trauma or surgery, malignencies should be excluded [1-3]. There was no causative factor for our patient's chylothorax except nausea and vomiting, and central subclavian catheterisation. Transthoracic echocardiography, oesophago-gastro-duodenoscopy and abdominal CT scan in our patient excluded malignancy or infection. Chest CT disclosed mediastinal enlargement and bilateral pulmonary microemboli. Further immunological studies of serum excluded possibility of SLE. On the other hand, fibrinogen, D-dimer, Factor VIII and IX (Christmas) levels were elevated. These parameters would elevate in most of conditions such as pulmonary thromboembolus, disseminated intravascular coagulation or septic shock. In our patient, there were bilateral pulmonary microemboli and mediastinal expansion on chest CT scan which could not be distinguished from abscess or haemorrhage. On the other hand she had a long line inserted into the right subclavian 
vein and through this line $3 \mathrm{H}$ treatment and hyperalimentation was continued. Central venous catheterisation may lead to venous thrombosis in the neck, and this procedure may obstruct the drainage of the chyle into the subclavian vein which will then result in uni-or bilateral chylothorax especially when venous entry is into the left subclavian vein. Even in such a case, a latency period of 2 to 7 days exist between the time of venous injury and chylothorax, and chyle accumulates first in the posterior mediastinum $[1,5]$. In the literature reported complications related to central venous catheterisation and hyperalimentation treatment are mediastinal haematoma and leakage of intravenous fluid because of the displacement of the tip of the central venous catheter $[6,7]$. Chest MR of our patient revealed persistent mediastinal enlargement releated with haemorrhage like mediastinal collection; and thrombus in the azygous vein. This condition could be explained by the central venous catheterisation procedure, however except the left inferior pulmonary artery and azygous vein, other mediastinal main vasculatures were all patent; and mediastinal enlargement could not be explained by haemorrhage, chylous fluid or intravenous fluid accumulation. Bipedal lymphangiography and/or lymphoscintigraphy has been recommended to clarify the cause and source of the leak of the chyle $[1,2]$. Also to demonstrate the aetiology of the mediastinal enlargement the mediastinal endoscopy could be done. However, we did not perform these radiologic or surgical procedures because of the lack of the time, and worse health condition of the patient. Also there was no postmortem investigation to demonstrate the thoracic duct and mediasten anatomy. On the other hand, in our knowledge assocation of intracranial aneurysm and chylothorax has not been reported in English medical literature. In patients with SLE, intracranial berry aneurysm and chylothorax have been reported to occur simultaneously [8, 9], but our patient did not have SLE.

Continued loss of proteins, immunoglobulins and lipids into the pleural spaces may lead to secondary immunodeficiency; and these may predispose the patient to opportunistic microorganisms such as Histoplasma spp. However, infection of chylous fluid itself is very uncommon because it is inherently bacteriostatic [1]. In our patient, chylous fluid cytology showed numerous leukocytes without atypical features and Gram (-) bacilli whose cultures revealed Acinetobacter calcoaceticus-baumanii complex. In the literature chylous fluid infected with different types of microorganisms were reported [1], but we were unable to cite Acinetobacter spp as the agent. It is not possible to conclude that chylothorax had been caused by, or contaminated with this microorganism because the blood cultures were negative for Acinetobacter spp. Besides the infection can be referred as nasocomial infection, when we take the resistance pattern of the microorganism into consideration.

\section{Conclusion}

As conclusion, sometimes the aetiology of chylothorax in adults may be uncertain and/or complicated; and in the treatment may also be difficult especially when it is contaminated with microorganisms. In such a case hypervolemia and hypertension may further aggravate the ventilation-perfusion of damaged lungs.

\section{References}

1. Nair SK, Petko M, Hayward MP. Aetiology and management of chylothorax in adults. Eur J Cardiothorac Surg. 2007;32(2):362-369.

2. Romero S. Nontraumatic chylothorax. Curr Opin Pulm Med. 2000;6(4):287-291.

3. Akcali O, Kiray A, Ergur I, Tetik S, Alici E. Thoracic duct variations may complicate the anterior spine procedures. Eur Spine J. 2006;15(9):1347-1351.

4. Kinnaert P. Anatomical variations of the cervical portion of the thoracic duct in man. J Anat. 1973;115(Pt 1):4552.

5. Beljaars GH, Van Schil P, De Weerdt A, Suys B, Wojciechowski M, Jorens PG. Chylothorax, an unusual mechanical complication after central venous cannulation in children. Eur J Pediatr. 2006;165(9):646-647.

6. Inaba K, Sakurai Y, Furuta S, Sunagawa R, Isogaki J, Komori Y, Uyama I. Delayed vascular injury and severe respiratory distress as a rare complication of a central venous catheter and total parenteral nutrition. Nutrition. 2009;25(4):479-481.

7. Sirmali M, Gezer S, Goktas U, Erturk H, Kaya S. A rare complication of subclavian vein catheterization: mediastinal haematoma, delayed resorption. Acta Chir Belg. 2005;105(3):324-325.

8. Lin YJ, Chen DY, Lan JL, Hsieh TY. Chylothorax as the initial presentation of systemic lupus erythematosus: a case report. Clin Rheumatol. 2007;26(8):1373-1374.

9. Sanchez-Ojanguren J, Matias J, Misis M, Olive A. Systemic lupus erythematosus, berry aneurysm and subarachnoid haemorrhage. Clin Rheumatol. 1999;18(2):165-166. 